\title{
Trends in Chronic Obstructive Pulmonary Disease Hospitalization Rates in Texas 2001-2009
}

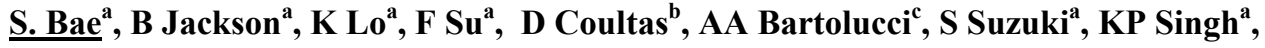 \\ ${ }^{a}$ Department of Biostatistics, University of North Texas Health Science Center School of Public Health; Fort \\ Worth, Texas \\ ${ }^{b}$ Department of Medicine, University of Texas Health Science Center at Tyler; Tyler, Texas \\ ${ }^{c}$ Department of Biostatistics, University of Alabama Birmingham School of Public Health; Birmingham, \\ Alabama \\ Email:sejong.bae@unthsc.edu
}

\begin{abstract}
Chronic Obstructive Pulmonary Disease (COPD) is a progressive lung disease associated with substantial morbidity and mortality. COPD exacerbations (worsening of symptoms) can lead to hospital admissions. One of the treatment goals in managing COPD is to reduce the exacerbation rates, since these rates affect the patient's quality of life, drive up treatment related costs, and if untreated could lead to decreased survival rate. A study of trends in COPD exacerbations is important for several reasons. First, by examining trends, we may determine whether recent treatment methods and disease relevant policies are effective. Second, by identifying disparities in exacerbation rates, health care professionals may be able to direct more efforts toward populations with the highest rates. Third, an analysis of trends will inform researchers on the progress of COPD research.
\end{abstract}

In this study we explored the change in race specific rates of COPD exacerbations according to county metropolitan status among the Texas population from 2001 to 2009

Keywords: Chronic Obstructive Pulmonary Disease (COPD), time trend, disparity 


\section{INTRODUCTION}

Chronic Obstructive Pulmonary Disease (COPD) is a progressive lung disease associated with substantial morbidity and mortality. COPD exacerbations (worsening of symptoms) can lead to hospital admissions. One of the treatment goals in managing COPD is to reduce the exacerbation rates, since these rates affect the patient's quality of life, drive up treatment related costs, and if untreated could lead to decreased survival rate (Wedzicha and Seemunga, 2007; Partridge et al., 2009). COPD patients have increased healthcare utilization relative to their non-COPD counterparts, in 2008 the estimated aggregate national inpatient costs for COPD was \$6.1 billion (Mapel 2000, Wier 2011). Between 1988 and 1999 the Center for Disease Control MMWR reported that nationally there was an increasing trend in the overall rate of COPD hospitalizations (Mannino et al., 2002). The highest hospitalization rates were among non-Hispanic (NH) White males.

A study of trends in COPD exacerbations is important for several reasons. First, by examining trends, we may determine whether recent treatment methods and disease relevant policies are effective. Second, by identifying disparities in exacerbation rates, health care professionals may be able to direct more efforts toward populations with the highest rates. Third, an analysis of trends will inform researchers on the progress of COPD research. Moreover, examination of hospitalization rates may offer a method for evaluating the effectiveness of various interventions. By analyzing the rates at the state and county level, locally tailored healthy policy may be implemented that could maximize health benefits and minimizing health inequality.

This study attempts to contribute to the knowledge base by exploring the change in race specific rates of COPD exacerbations according to county metropolitan status among the Texas population between the years 2001 to 2009

\section{METHODS}

\subsection{Data Source}

The data were obtained from the Texas Health Care Information Council (THCIC) discharge datasets between 2001 and 2009 (Texas Hospital Inpatient Discharge Public Use Data File. Texas Department of State Health Services, Center for Health Statistics, Austin, Texas, 2010). These were publicly available data files, which included patient demographics including age group, gender, race and ethnicity, as well as geographic data, diagnoses and procedures and payment methods. Population estimates were obtained from the Texas state demographer's website (http://txsdc.utsa.edu/tpepp/txpopest/php accessed 15 December, 2009) for all years 2001-2009. The estimates reported were stratified by gender, race and age across all counties in Texas and were projected based off of the 2000 census data to take into account annual deaths and growth changes. The THCIC data was combined with the demographer's data to create the analysis dataset. Urban Influence codes were used to categorize all of the 254 counties in Texas as either Metropolitan or Non-Metropolitan.

\subsection{Variable Definitions}

For the analysis, the age of subjects was restricted to individuals over the age of 18 . Race/Ethnicity was categorized and restricted to: Non Hispanic (NH) White, NH Black, and Hispanic. Hospitalizations due to COPD were determined by the Agency for Healthcare Research and Quality's prevention indicator technical specifications

(http://www.qualityindicators.ahrq.gov/Modules/PQI TechSpec.aspx, Accessed 15 December, 2009) which used the admitting diagnosis ICD-9 codes 491.xx (chronic bronchitis), 492.xx (Emphysema), and 496.xx (chronic airway obstruction not elsewhere classified) and their respective subcategories. Admitting diagnosis was chosen to estimate severe COPD exacerbations which require 
hospitalization; the use of ICD-9 codes for COPD classification has been verified in other studies (Kabir et al., 2010; Arbex et al., 2009; Mapel et al., 2000)

\subsection{Outcome Definition}

Category specific hospitalization rates were calculated as the frequency of exacerbations in a category divided by the projected population at risk for that category, per 100,000 populations. The rates across gender and race of patients were calculated according to metropolitan status.

\subsection{Statistical Analysis}

Poisson regression was used to explore the change in rates of COPD hospitalization over time, as well as across categories. Based on our previous work, we checked for the interaction between metropolitan status and time, and found it to be significant. Therefore we performed stratified analyses for both rural and urban exacerbations (Jackson et al., 2011). The analysis was performed using SAS version 9.2 software (SAS Institute Inc. Cary, NC, USA). All results were considered statistically significant if the p-value was less than 0.05 .

\section{RESULTS}

In 2001 and 2009, females had higher hospitalization rates than males across all race categories and in both metropolitan and non-metropolitan counties (Table 1). The rates of non-metropolitan residents were higher than metropolitan residents for all gender and race combinations. Over the nine years analyzed, all county type, race, and gender combinations experienced a decrease in the percentage change from 2001. The hospitalization rates with the lowest percent change over time period analyzed were for $\mathrm{NH}$ males in Non-metropolitan and Metropolitan counties $(8.91 \%$ and 9.29\%). Metropolitan Hispanic males and females had the largest percent decrease (44.78\% and $46.51 \%$ ) while in Non metropolitan counties, NH Black females and males had the largest percent decrease $(27.11 \%$ and $32.26 \%)$.

In the multivariable adjusted poisson regression models (Table 2), gender, race, and age category were all significant. There was significant interaction between year and metropolitan status $(\mathrm{p}<0.0001$, data not shown) therefore models 2 and 3 were run for Non-metropolitan and Metropolitan counties respectively. These stratified regressions showed that only in metropolitan counties was there a significant difference between genders. For both models the estimate for time showed a significant decrease for the effect of time (Non Metropolitan Rate Ratio $=0.97, \mathrm{p}<0.0001$; Metropolitan Rate Ratio=0.96, $\mathrm{p}<0.0001$ ). NH Whites had the highest hospitalization rate, intermediate for NH blacks, and lowest for Hispanics.

\section{DISCUSSION}

COPD hospitalization rates in both metropolitan and non-metropolitan Texas counties declined over the period 2001 to 2009, in contrast to an increase nationally from 1988 to 1999 . Moreover, females had higher hospitalization rates compared to males. A number of factors may have contributed to these trends including decline in prevalence associated with decline in smoking and other risk factors, improved access to health care, and changes in medical practice patterns and medical management. Analysis of the COPD hospital discharge rates using data from the Dartmouth Atlas project revealed that the annual Texas average discharges for COPD per 1,000 Medicare enrollees was predominantly higher than the national average between 1996 and 2005 (http://www.dartmouthatlas.org/data/table.aspx?ind=62, Accessed 12, June, 2010). The discharge rates are much higher than the rates observed from the THCIC data; this may indicate that the decreasing trend in COPD hospitalizations could result from an increase in outpatient utilization. However, it should be noted that the Dartmouth project's results are based off of Medicare patients, 
this older population has a much higher prevalence of COPD than younger age groups. The lower hospitalization rates observed from the NH black and Hispanic populations could be due to a lower prevalence of COPD or under utilization of inpatient services (Laditka, 2006). The anticipation of health care costs may prevent certain populations from seeking health services in the event of an exacerbation. However the decline in hospitalizations was observed across all racial and ethnic groups. Similarly, the gender differences observed may be attributable to different patterns of health care utilization.

The rate differences between metropolitan and non-metropolitan counties was previously observed in a cross sectional analysis of the THCIC data (Jackson et al. 2011), however this research shows that rates differ across time. The degree of rurality has been found to be associated with increased mortality of COPD (Abrams et al., 2011) as well as increased hospitalization rate for all ambulatory care sensitive conditions (Laditka, 2009). This trend analysis provides a dynamic view of how COPD hospitalizations have changed over recent years in Texas. The overall decline in hospitalization rates in Texas is similar to the trend observed in Massachusetts (Kabir et al., 2010). Moreover, the national annual estimated rate of office visits with COPD as the first listed diagnosis shows a decrease around the year 2000, for both males and females as well as for NH blacks according to the MMWR for COPD (Mannino et al., 2002).

There are a few limitations of this study. First, this analysis uses publicly available administrative data files which contained limited information, and variables on smoking, spirometry, and medications were not provided. There have been numerous clinical trials on the efficacy of various medications, and the role of smoking in COPD has been well documented. Patient's use of these medications could have contributed to the decline in hospitalizations. In addition to this, there were no unique patient identifiers, and repeated visits could have introduced bias to the results. Second, the analysis included patients who were under the age of 40 who may have been misdiagnosed with COPD; however, this subgroup made up a small proportion of the sample size each year and their inclusion in the analysis likely had limited effect on the findings. Third, this study has potential for ecologic fallacy. The unit of analysis was at the county level, where varying geographic factors such as prevalence of smoking, air pollution, access to care, and occupational exposures are different across locations. Therefore the frequency of exposure and proximity to sources of pollution could have affected the exacerbation rates, as well as variations in patterns of medical treatment. Due to these limitations, further studies are needed to explain any spatiotemporal changes in the variations of COPD hospitalization rates between racial and ethnic groups.

Exacerbations of COPD can be prevented and treated. The results of this analysis show that there is an overall decreasing trend across the nine year period of COPD hospitalization rates in Texas. The findings show that NH blacks have a lower hospitalization admission rate than NH whites, and that the rates differ according to metropolitan county status. Race-wise differences suggest lower prevalence of COPD, less access to hospital services, or decreased susceptibility to COPD exacerbations. By addressing these disparities, progress can be made to decrease healthcare utilization among COPD patients and to improve their quality of life.

\section{ACKNOWLEDGEMENTS}

Completion of the manuscript was funded, in part, by a grant from the National Heart, Lung, and Blood Institute of NIH, Grant number R18 HL092955.

\section{REFERENCES}

Wedzicha JA, Seemunga TA (2007) COPD exacerbations: defining their cause and prevention. Lancet, 370 , 786-796.

Partridge MR, Schuermann, W, Beckman Ola et al. (2009) Effect on lung function and morning activities of budesonide/formoterol versus salmeterol/fluticasone in patients with COPD. Therapeutic advances in Respiratory Disease, 3,147-157. 
Mapel DW, Hurley JS, Frost FJ, et al (2000) Health Care Utilization in Chronic Obstructive Pulmonary Disease. Arch Intern Med ,160, 2653-2658.

Wier LM, Elixhauser A, Pfuntner A, Au DH (2011) Overview of hospitalizations among patients with COPD, 2008: Statistical Brief \#106. Healthcare Costs and Utilization Project (HCUP)

Mannino DM, Homa DM, Akinbami LJ et al. (2002) Chronic Obstructive Pulmonary Disease Surveillance; United States, 1971-2000. MMWR Surveill Summ, 51,1-16

Texas Hospital Inpatient Discharge Public Use Data File [2001-2009]. Texas Department of State Health Services, Center for Health Statistics, Austin, Texas. [2010];

Texas State Data Center and Office of the State Demographer. Texas population estimates program. [retrieved from http://txsdc.utsa.edu/tpepp/txpopest.php;2009]

Prevention Quality Indicators Technical Specifications [http://www.qualityindicators.ahrq.gov/Modules/PQI TechSpec.aspx]

Kabir Z, Connoly, GN Koh, Clancy L (2010) Chronic obstructive pulmonary disease rates in Massachusetts: a trend analysis. $Q J \mathrm{Med}, 103,163-168$.

Arbex M, de Souza Conceicao G, Cendon S, et al (2009) Urban air pollution and chronic obstructive pulmonary related disease-related emergency department visits. Journal of Epidemiology \& Community Health ,63,777-783

Jackson BE, Suzuki S, Lo K et al. (2011) Geographic disparity in COPD hospitalization rates among the Texas population. Respir Med, 105(5), 734-739

SAS v9.2, SAS Institute Inc. Cary, NC, USA.

Laditka JN, Laditka SB (2006) Race, ethnicity and hospitalization for six chronic ambulatory care sensitive conditions in the USA. Ethn Health 11(3): 247-263

Abrams TE, Vaughan-Sarrazin M, Fan VS, Kaboli PJ (2011) Geographic Isolation and the Risk of Chronic Obstructive Pulmonary Disease-Related Mortality. Annals of Internal Medicine, 155(2) 80-86

Laditka JN, Laditka SB, Probst JC (2009) Health care access in rural areas: evidence that hospitalization for ambulatory care-sensitve conditions in the United States may increase with the level of rurality. Healthy Place 15(3): 731-740

\section{TABLES}

Table 1 COPD hospitalization rates stratified by metropolitan status, gender and race.

\begin{tabular}{lllccc}
\hline & & 2001 & 2009 & $\%$ change \\
\hline Non Metropolitan & \multirow{2}{*}{ NH White } & Female & 389.72 & 342.66 & $12.07 \%$ \\
& & Male & 329.96 & 300.56 & $8.91 \%$ \\
& \multirow{4}{*}{ NH Black } & Female & 255.69 & 186.37 & $27.11 \%$ \\
& Hispanic & Female & 200.83 & 136.04 & $32.26 \%$ \\
& & 137.42 & 100.16 & $27.11 \%$ \\
Metropolitan & NH White & Female & 121.92 & 90.41 & $25.85 \%$ \\
& \multirow{4}{*}{ NH Black } & Male & 239.15 & 289.68 & $11.21 \%$ \\
& & Female & 185.86 & 156.85 & $9.29 \%$ \\
& Hispanic & Male & 173.87 & 137.61 & $20.85 \%$ \\
& & Female & 90.13 & 48.21 & $46.51 \%$ \\
All Texas & & 86.54 & 47.78 & $44.78 \%$ \\
\hline
\end{tabular}

Rates reported per 100,000 observations; NH- Non Hispanic

Data came from the Texas Healthcare Information Council 2001-2009. 
Table 2 Rate Ratios for the different Poisson regression models

\begin{tabular}{|c|c|c|c|c|c|c|c|}
\hline \multirow[b]{2}{*}{ Variables } & & \multicolumn{2}{|c|}{ Model 1} & \multicolumn{2}{|c|}{ Model 2: Non Metro } & \multicolumn{2}{|c|}{ Model 3: Metro } \\
\hline & & $\mathrm{RR}$ & $\mathrm{p}$-value & $\mathrm{RR}$ & $\mathrm{p}$-value & $\mathrm{RR}$ & $\mathrm{p}$-value \\
\hline Gender & Female vs Male & 1.07 & $<0.0001$ & 0.99 & 0.5907 & 1.08 & $<0.0001$ \\
\hline \multirow[t]{3}{*}{ Race } & NHB vs NHW & 0.88 & $<0.0001$ & 0.86 & $<0.0001$ & 0.87 & $<0.0001$ \\
\hline & H vs NHW & 0.45 & $<0.0001$ & 0.57 & $<0.0001$ & 0.43 & $<0.0001$ \\
\hline & NHO vs NHW & 1.20 & $<0.0001$ & 5.31 & $<0.0001$ & 1.03 & 0.2885 \\
\hline \multirow[t]{2}{*}{ Age category } & $45-64$ vs $18-44$ & 18.38 & $<0.0001$ & 17.08 & $<0.0001$ & 18.39 & $<0.0001$ \\
\hline & $65+$ vs $18-44$ & 95.97 & $<0.0001$ & 65.04 & $<0.0001$ & 102.82 & $<0.0001$ \\
\hline Metropolitan Status & Non Metro vs. Metro & 1.00 & 0.6037 & & & & \\
\hline Year & & 0.96 & $<0.0001$ & 0.97 & $<0.0001$ & 0.96 & $<0.0001$ \\
\hline
\end{tabular}

NHB-Non Hispanic Black, NHW-Non Hispanic White, NHO-Non Hispanic Other, H-Hispanic, RR- Rate Ratio

Model 1 adjusts for Gender, Race, Age Category, Metropolitan Status, and Year

Models 2 and 3 adjust for Gender, Race, Age Category, and Year 